\title{
Biogenic synthesis of silver nanoparticles using pigment producing Streptomyces sp and Its drug binding assay
}

\author{
Santhanalakshmi Krishnamoorthy ${ }^{{ }^{*} \text {, Natarajan Ekambaram }}{ }^{2}$ \\ ${ }^{1,2}$ Department of Biotechnology, National College (Autonomous), Tiruchirappalli, India. \\ *Corresponding Author: santhuviro@gmail.com
}

Available online at: www.isroset.org

Received: 03/Oct/2018, Accepted: 20/Oct/2018, Online: 31/Oct/2018

\begin{abstract}
Cardiovascular disease, management of hypertension is mostly treated by Amlodipine, atorvastatin and telmisartan. Amikacin also one of the pharmaceutical drugs against bacterial infections. The Streptomyces $s p$. DSK2 melanin absorbs the drugs in different concentration, different temperature and different incubation timings. The result shows that the Streptomyces sp. DSK2melanin absorb the drugs in $40^{\circ} \mathrm{C}$ and the reaction timings were $180 \mathrm{~min}$. Amlodopine bind strongly at $40{ }^{\circ} \mathrm{C}$ at 180 min, other two drugs Atorvastatin and Amikacin moderate bind at $40{ }^{\circ} \mathrm{C}$ at $120 \mathrm{~min}$. Other biomedical studies of the Streptomyces sp. DSK2 melanin silver nanoprticle was synthesized in 180 s by the microwave radiation method. The silver nanoparticle range $50 \mathrm{~nm}$ in size, which is confirmed by TEM analysis, UV spectrum at 420nm , FTIR Spectrum , XRD. The melanin showed the potent antioxidant activity was determined by DPPH assay, the maximum activity was 87.96 and the total antioxidant activity phosphomolybdate assay) was 88.05. in this study, Streptomyces sp. DSK2 Melanin has great biomedical activity.
\end{abstract}

Keywords: Drug, Streptomyces sp., Melanin, microwave radiation, silver nanoparticles

\section{INTRODUCTION}

Melanin is nearly a ubiquitous pigment. Melanins are synthesized from many living organisms, both Prokaryotes and Eukaryotes. Melanins are negatively charged, hydrophobic in nature and high-molecular-weight compounds. The formation of melanin controlled by tyrosinase and 4-hydroxyphenylacetic acid hydroxylase, which converts tyrosine to dopa and then to dihydroxyindole and dihydroxyindole-2-carboxylic acid, by a series of enzymatic and non-enzymatic reactions. Many microorganisms produced melanin physico-chemical characteristics were studied by various methods and biological roles and functions of melanin was acting as powerful antioxidants via, for example, metal sequestration. The amino, carboxyl, phenolic and hydroxyl group of melanin binding sites for drugs [1] and metal ions [2]. Microbial melanin have a great potential applications in, cosmetics, and pharmaceutical industries and agriculture field. Many researchers revealed the streptomyce melanin have a good photoprotectant and the best mosquitocidal activity of Bacillus thuringiensis subsp. israelensis [3]. A natural and synthetic melanin affinity study was demonstrated by many researchers and they applied in various drugs in both in vitro and in vivo studies. Melanin containing tissues are accumulating and retain theses drugs is remarkable [4]. The accumulation and retention of drug residues in the pigmented tissues of the eye is widely acknowledged [5]. The basic drugs chloroquine and procaine penicillin (Atlasik et al. 1980), chlorpromazine [6], amphetamine, atropine and cocaine [7], binding affinity of the melanin was good. Melanin binding of substances in external and internal tissues (skin, hair, ear, eye, and brain) that (exogeneous and endogeneous) substances involved in pathogenetic effects onto the organism involved to analyse the long term disease associated with long-term therapy with a number of drugs [8]. Chlorpromazine binding to and subsequent release from retina melanin [9]. The microbial melanin binding with metal ions and various drugs more important characteristic of this pigment. The main functions of the metal ions can be act as cofactors of enzymes, catalyzing basic functions like electron transport, redox reactions and energy metabolism, and are essential for maintaining the osmotic pressure of the cell [10]. Atorvastatin is belongs to the group of "statins" drug mainly used to maintain the proper diet and reduce the cholesterol fats (such as LDL, triglycerides) and increase the good cholesterol in blood, simultaneously reduce the cholesterol in liver and inhibiting HMG-CoA reductase, an enzyme found in liver tissue, that plays a key role in the production of cholesterol in the body [11]. It also prevents heart from heart attacks and stroke. Telmisartan is also called as "Angiotensin receptor blockers (ARBs)". It also used to control the high blood pressure (hypertension) and kidney problems, edema (swelling of arms, legs, lips, tongue, or throat, the latter leading to breathing problems), and allergic reactions [12]. It may also helps to relax the blood vessels so blood can flow more easily. 
Amlodipine is a calcium channel blocker that dilates (widens) blood vessels and improves blood flow. Amlodipine is used to treat chest pain (angina) and other conditions caused by coronary artery disease. Amlodipine is also used to treat high blood pressure (hypertension), relaxing the smooth muscle in the arterial wall, decreasing total peripheral resistance and hence reducing blood pressure. In angina it increases blood flow to the heart muscle [13]. Amikacin is an aminoglycoside antibiotic which is treating the certain serious bacterial infections. It works by inhibiting the production of bacterial proteins, which causes bacterial cell death. Amikacin, kanamycin and tobramycin stable complex studies are demonstrated with synthetic melanin in invitro [14] and the modified amikacin and tobramycin interaction with melanin was followed with $\mathrm{Cu} 2+$ and $\mathrm{Zn} 2+$ ions [15].

Nanobiotechnology is a multidisciplinary field and immensely developing the field such as biotechnology, nanotechnology, physics, chemistry, and material science [16], as it wide-ranging applications in different areas of science and technology. Many researchers synthesized a nanoparticles based on chemical, physical and biological method. Biogenic based nanoparticles synthesis using the like bacteria [17] fungi [18], actinomycetes [19]lichens [20] algae [21], etc. Biogenic nanoparticles are environment friendly, as no toxic chemical is involved in synthesis, and also the synthesis process takes place at ambient temperature and pressure conditions. So many researchers focusing the biogenic nanoparticle synthesis nanoparticles [22].Water soluble melanins have great potential applications such as protect a skin from UV radiation and used in in paints, varnishes and other surface protection formulations. Water soluble melanin is used in solid plastic films of polyvinyl alcohol to be employed in conjunction with other plastics to make laminated sheets or lenses. Soluble melanin binds a number of chemicals and drugs, thereby making it useful in the detection of low levels of compounds and metabolites, or in the elimination of toxic target. Streptomyces lusitanus and Bacillus thuringensis based water soluble melanin from biogeneic nanoparticles especially silver nanoparticles were exploited for their novel and wide range of potential biomedical applications, because of their unique and distinct physical, chemical and biological properties. For pigment based silver nanoparticle synthesis Microwave irradiation provides increases the rapid synthesis and generating the uniform non aggregated nanoparticles. This applicable method useful to study the biological ligands and more usage in biomedical applications, biological reducing agents were realized as new materials and functional groups. In the present investigation followed the binding studies of Streptomyces DSK2 melanin in pharmaceutical drugs in different temperature, different time intervals and different concentrations. Melanin from Streptomyces DSK2 binds to the atorvastatin, telmisartan, Amlodipine and Amikacin adsorption studies of drug molecules. Another investigation biogeneic based active silver nanoparticle synthesis in microwave assisted method. So the current study Streptomyces DSK2 produced pigment based silver nanoparticles (AgNPs) which are potentially been exploited in medicine for antibacterial, antifungal activity.

\section{MATERIALS AND METHODS}

\section{Extraction and purification of melanin pigment from Streptomyces DSK2}

Streptomyces sp. DSK2 was grown in Tyrosine starch casein agar medium with $\mathrm{pH}$ 7.2. Streptomyces sp. DSK2 was sequence was submitted in NCBI (Genebank accession No.KU174534).The medium was autoclaved at 15 psi $\left(121^{\circ} \mathrm{C}\right)$ for $20 \mathrm{~min}$. After the culture was inoculated and incubated at $37 \circ \mathrm{C}$ for $7-14$ days. After pigmentation the culture was filtered by filter paper remove the myceliums. Then the filtrate was adjusting with $0.1 \mathrm{~N} \mathrm{Hcl} \mathrm{pH} \mathrm{3.5,} \mathrm{add}$ $0.1 \mathrm{~N} \mathrm{Nacl} \mathrm{pH}$ 7. The pigment was separated by centrifugation at $8,000 \mathrm{~g}$ and it was extracted, purified for further process. The purified melanin pigment was used for adsorption studies.

\section{Conformation of Melanin production}

The Melanin conformation was tested by Streptomyces $s p$. DSK2 inoculated tyrosine starch casein broth taken and it was filtered. The $2 \mathrm{ml}$ of filtrate was culture and $1 \mathrm{ml}$ of $0.4 \%$ substrate solution (L-Tyrosine (or) L-dopa). The reaction mixture was incubated at $37 \mathrm{oC}$ for $30 \mathrm{~min}$ for Ltyrosine and 5 min for L-Dopa and red coloration resulting from dopachrome formation was observed and read using spectrophotometer at $480 \mathrm{~nm}$. When there was no coloration within these periods, the reaction mixture was further incubated for as long as 2 hrs. After incubation melanin was then formed within $30 \mathrm{~min}$ as discuessed in previous literature [23].

\section{Melanin - Drug binding assay}

Sterptomyces sp. DSK2 melanin pigment $(1 \mathrm{mg} / \mathrm{ml})$ was dissolved in sodium phosphate buffer of $\mathrm{pH} 7.2$ and drug solutions (atorvastatin, telmisartan, Amlodipine and Amikacin) were added and to make a final volume of $2 \mathrm{ml}$. Initial concentrations of drugs ranges from 10 to $50 \mu \mathrm{g}$ for atorvastatin, 30-150 $\mu \mathrm{g}$ for telmisartan, 7-35 $\mu \mathrm{g}$ for amlodipine and 10 to $50 \mu \mathrm{g}$ for Amikacin with a common buffer. Control was used as melanin pigment. The samples were incubated at different time intervals (10-30min) and different temperature ranges $\left(20-60^{\circ} \mathrm{C}\right)$. The binding drugs with quantity were measured by spectrophotometrically at 245nm, 210nm, 370nm and 270nm respectively. Melanin bound with drugs were calculated by the difference between the initial drug to melanin and the amount of unbound drug, and bound drug per $1 \mathrm{mg}$ melanin

\section{Absorption studies of Melanin -Drug binding assay}

The adsorption of drug to Streptomyces sp. DSK2 melanin was prepared in $\mathrm{pH} 7.2$, temperature at $37{ }^{\circ} \mathrm{C}$ and increasing 
drug concentrations. Melanin pigment $(1 \mathrm{mg} / \mathrm{ml})$ was taken and different drug solution was kept $3 \mathrm{hr}$ and incubated at 37 ${ }^{\circ} \mathrm{C}$. After the drug melanin complex was centrifuged and the supernatant was taken for adsorbed drugs was calculated.

\section{Effect of concentrations of drugs on uptake by melanin pigment}

The experiments were conducted in test tubes containing various concentrations of drugs (10 to $50 \mu \mathrm{g}$ for atorvastatin, 30-150 $\mu \mathrm{g}$ for telmisartan and 7-35 $\mu \mathrm{g}$ for amlodipine and 10 to $50 \mu \mathrm{g}$ for Amikacin) in common buffer. $1 \mathrm{mg} / \mathrm{ml}$ of Streptomyces sp. DSK2 melanin pigment was mixed with drugs. The maximum absorbance was measured at $245 \mathrm{~nm}$, $210 \mathrm{~nm}, 370 \mathrm{~nm}$ and $270 \mathrm{~nm}$.

\section{Effect of temperature of drugs on uptake by melanin pigment}

The optimum temperature ranges were followed up to 20-

$60 \circ \mathrm{C}$. The drugs concentrations ranges are same followed in effect of concentration experiment. The maximum absorbance was measured.

\section{Effect of time of drugs on uptake by melanin pigment} The effect of time duration which could be reacts into the melanin pigment. various time duration ranges (30-180min) were followed in these reactions and the maximum absorbance was measured.

\section{Synthesis of pigment based biogeneic Silver Nanoparticles} The Sterptomyces sp. DSK2 melanin was used to synthesis of silver nanoparticles from silver nitrate facilitated by microwave radiation method. different concentration of silver nanoparticles $(2,4,6,8$ and 10) of $1 \mathrm{~mL}$ silver nitrate solutions were added in to $1 \mathrm{~mL}$ of $0.1 \mathrm{mg} / \mathrm{mL}$ melanin. The mixture solution was incubated in a microwave $(2.45 \mathrm{GHz}$ frequency) oven. Different time reaction for synthesis of melasnin based silver nanoparticles was observed at $10 \mathrm{~s}, 30 \mathrm{~s}$, 60s, 90s, 120s, and 180s. The silver nanoparticle was confirmed by UV spectrometer, FTIR, XRD and TEM analysis. The UV absorption spectrum of silver nanoparticle was analysed between 300-600 nm.

\section{DPPH Radical Scavenging Activity}

Various concentrations of melanin silver nanoparticle (100, 200,400 and $800 \mu \mathrm{g} / \mathrm{mL}$ ) was mixed with $500 \mu \mathrm{L}$ of $99.5 \%$ ethanol and $125 \mu \mathrm{L}$ of $0.02 \% \mathrm{DPPH}$ in $99.5 \%$ ethanol. The mixture was incubated at $30{ }^{\circ} \mathrm{C}$ in dark for $30 \mathrm{~min}$. The reduction of DPPH was measured at $517 \mathrm{~nm}$ using UV spectrophotometer and the percentage inhibition of the DPPH radical was calculated. A lower absorbance of the reaction mixture indicates a higher DPPH radical scavenging activity. Ascorbic acid was used as a standard.

\section{Total Antioxidant Capacity of AgNo3 (Phosphomolybdate} Assay)

The Sterptomyces sp. DSK2 melanin silver nanoparticles total antioxidant capacity was determined by Phosphomolybdate Assay. 0.1 mL of at various concentrations (100, 200, 400,
800 and $1000 \mathrm{lg} / \mathrm{mL}$ ) of melanin silver nanoparticle was mixed with $1 \mathrm{~mL}$ reagent solution $(0.6 \mathrm{M}$ sulphuric acid, 28 $\mathrm{mM}$ sodium phosphate and $4 \mathrm{mM}$ ammonium molybdate) and incubated in a water bath at $95^{\circ} \mathrm{C}$ for $90 \mathrm{~min}$. The UV-Vis absorbance of the mixture at $30 \circ \mathrm{C}$ was recorded at $765 \mathrm{~nm}$ against a blank. $1 \mathrm{~mL}$ reagent solution and appropriate volume of the solvent was act as a blank. The antioxidant capacity was estimated using ascorbic acid as standard.

\section{RESULTS AND DISCUSSION}

Melanin contains carboxylic acid, amino, and hydroxy functional groups for chelating metal ions chemically. The Sterptomyces sp. DSK2 melanin bound to drugs was compared. The UV-Visible spectra of the drug bound melanin measured at $245 \mathrm{~nm}, 210 \mathrm{~nm}, 370 \mathrm{~nm}$ and $270 \mathrm{~nm}$ respectively, for telmisartan, atorvastatin, amlodipine and Amikacin. The basic compound with coplanar aromatic, indole fused rings (telmisartan), basic aromatic compound (amlodipine), and an aromatic imidazole compound (atorvastatin) and Amikacin (Fig.1) were dissolved in common buffer.

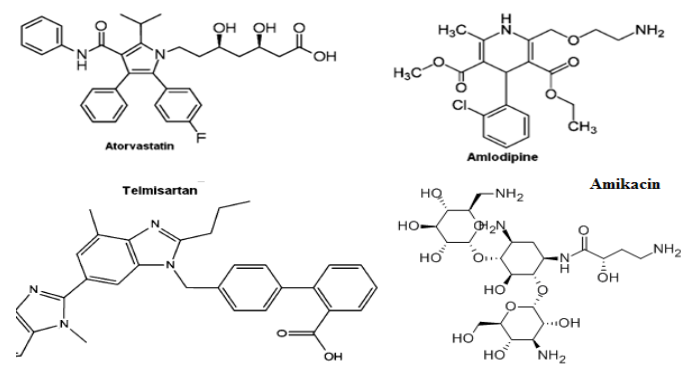

Figure.1 Drugs Strcture's

The optimization process very useful to analyses the different experimental conditions. The effect of different concentrations of drugs was measured at various UV absorption spectra. The maximum UV spectra range was obtained in Amlodipine. This drug bound to Sterptomyces sp. DSK2 melanin gives the maximum spectrum ranges (Fig.2).

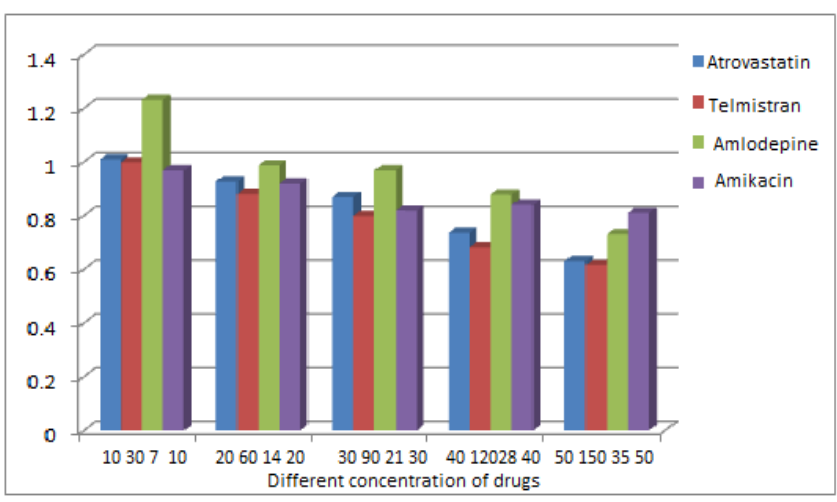

Figure.2 Different concentrations of drugs with melanin 
The effect of temperature on the adsorption of melanin pigment bound with drugs was investigated in various temperature ranges. Fig. 3 shows the drug concentrations were increased and same time temperature was also increased. The maximum temperature was observed in $40^{\circ} \mathrm{C}$. The adsorption of drugs on to melanin pigment is mainly due to the presence of charged functional groups like $-\mathrm{CH},-\mathrm{NH},-\mathrm{CO}$ and $-\mathrm{OH}$ [25], reported that the increase in the binding rates of bunazosin, dorzolamide, and timolol to synthetic melanin, drugs reach a plateau after $30 \min [26]$. Other investigation proceeding was time duration of binding capacity of the Streptomyces sp. DSK2 melanin pigment. Various time durations were followed. Fig.4 showed the maximum time duration was $180 \mathrm{~min}$. The pharmaceutical drugs that have the tendency to bind to melanin pigment. These screening works conclude the Streptomyces $s p$. DSK2 melanin pigment bound the pharmaceutical drugs which prove the significant. The applications of this pigment are seen in sunscreen lotions, antioxidants and other pharmaceutical uses as a drug carrier.

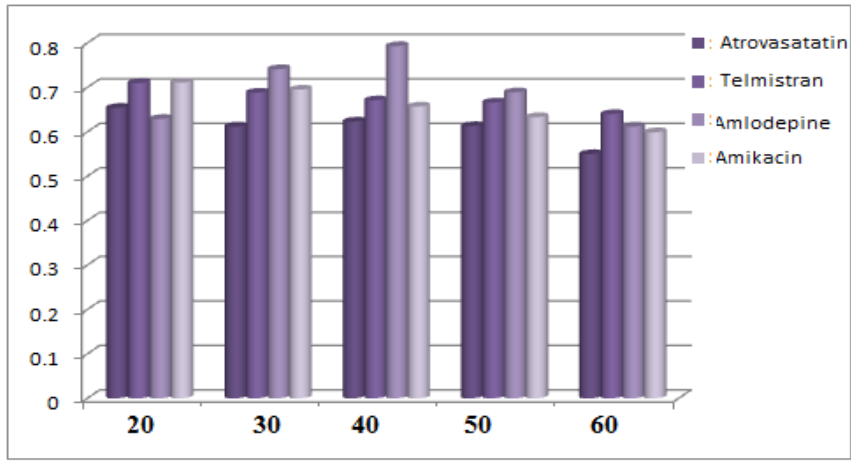

Figure.3 Different temperature of drugs with melanin

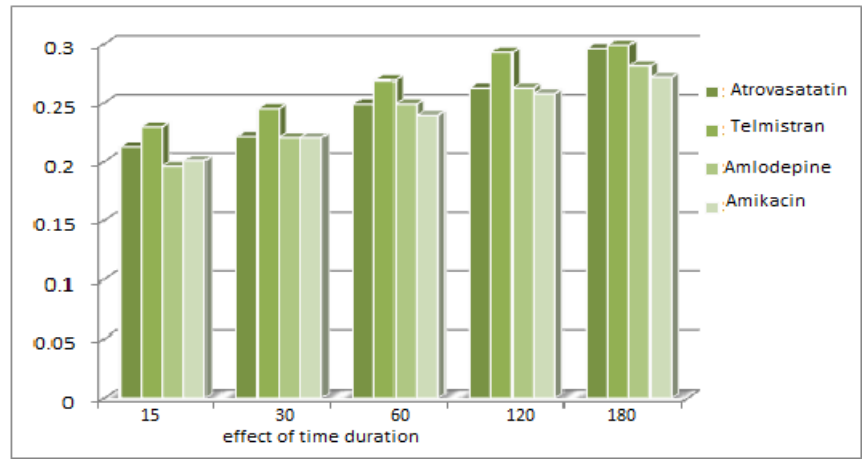

Figure.4 Different time duration of drugs with melanin

\section{Synthesis of Silver Nanoparticles}

The silver nanoparticle synthesis was basically consists various chemical and biological methods. The Streptomyces sp. DSK2 melanin pigment based silver nanoparticles were assisted by microwave radiation method[27]. The silver nanoparticles colour intensity was developed in different concentrations and different timing. The Streptomyces sp. DSK2 melanin bound silver nanoparticles (2mM) (Fig. 5a) maximum synthesis was confirmed by UV spectrophotometer at 420nm (Fig.5b \& 5c). Many silver nanoparticle have a excellent pharmaceutical applications.

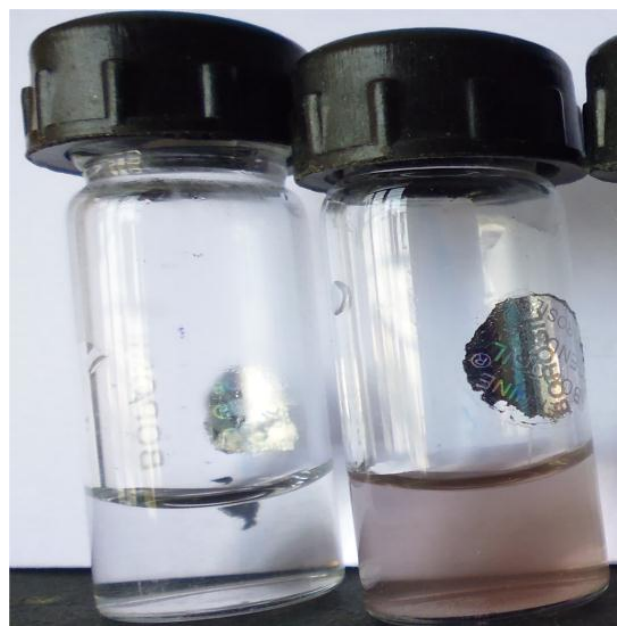

Fig.5a Streptomyces sp. DSK2 melanin based silver nanoparticle $(2 \mathrm{mM})$

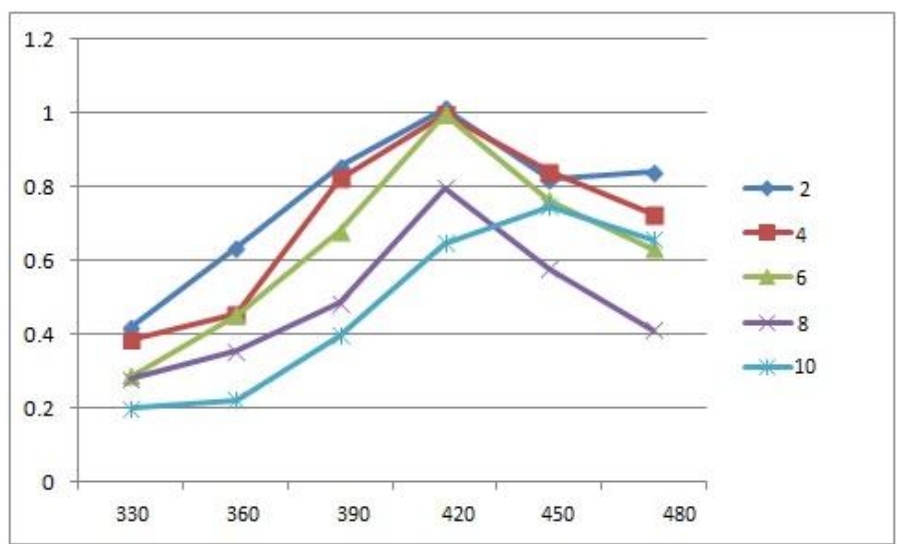

Fig.5b Streptomyces sp. DSK2 melanin based silver nanoparticle UV spectrum

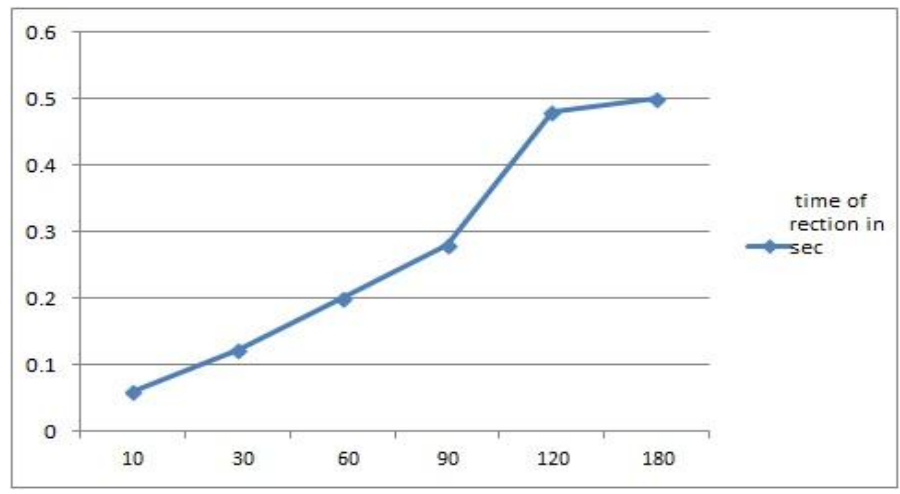

Fig.5c Streptomyces $s p$. DSK2 melanin based silver nanoparticle synthesis in 180s 


\section{Characterization of Silver Nanoparticles}

The Streptomyces $s p$. DSK2 melanin based silver nanoparticle maximum size was $50 \mathrm{~nm}$ by microwave radiation method. The size of the silver nanoparticle was measured by TEM analysis (Fig.6).

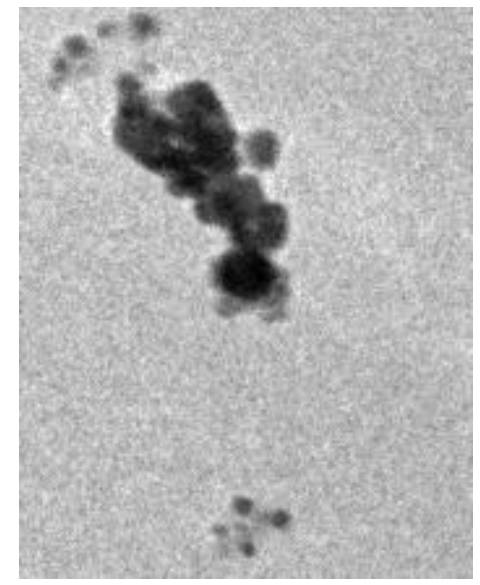

Fig.6 Streptomyces sp. DSK2 melanin based silver nanoparticle TEM analysis.

The presence of silver nanoparticles bound with melanin, FTIR spectrum (Fig.7) was confirms the functional groups were present. The absorbance at 3468,2374,1409,1020,663 due to $\mathrm{O}-\mathrm{H}$ stretches alcohols, phenolics and carboxylic acid, $\mathrm{CH}$ bend alkanes, $\mathrm{CN}$ stretches aliphatic amines and $\mathrm{CBr}$ stretches alkyl halides.The XRD analysis (Fig. 8) reveals the crystalline nature of silver nanoparticles with the intense peaks corresponding to $111,200,220$ and $3112 \mathrm{~h}$ values. These intense peaks were indexed based on the Face Centered Cubic structure of silver nanoparticles confirming the crystalline nature.

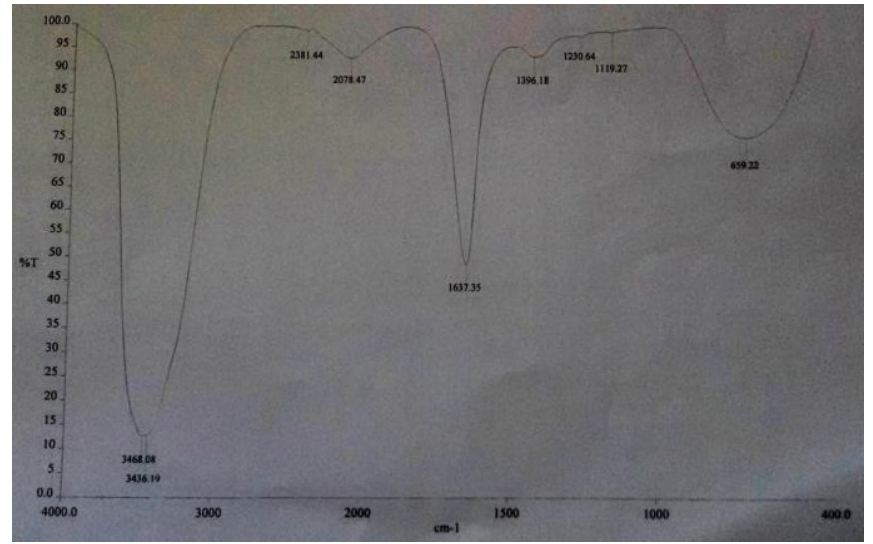

Fig.7 FTIR spectrum of Streptomyces sp. DSK2 melanin based silver nanoparticle

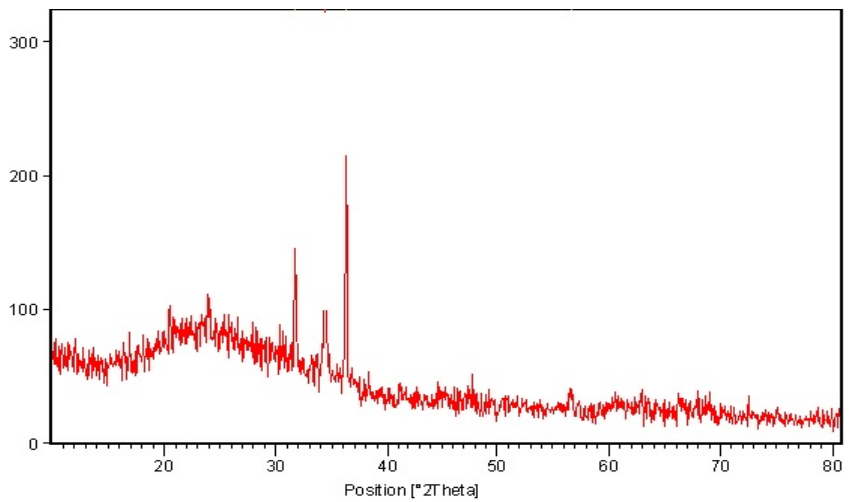

Fig.8 Streptomyces sp. DSK2 melanin based silver nanoparticle XRD analysis

The Streptomyces sp. DSK2 melanin based silver nanoparticle antioxidant activity was assessed by DPPH method (Fig.9). Different concentration of silver nanoparticles determined the DPPH radical scavenging activity. The DPPH maximum activity was $87.96 \mu \mathrm{g} / \mathrm{ml}$. The total antioxidant activities of silver nanoparticles were determined by this assay. The melanin silver nanoparticle concentration increases the total antioxidant activity was increased. The effective total antioxidant activity melanin silver nanoparticle concentration was $88.05 \mu \mathrm{g} / \mathrm{ml}$.

\section{CONCLUSIONS}

Sterptomyces sp. DSK2 melanin is a phenolic polymer and have broad spectrum of biomedical field [28]. The melanin based silver nanoparticle synthesis was assisted by microwave radiation method and attained in 180s. The size of the nanoparticle ranges were $50 \mathrm{~nm}$ which confirmed by TEM analysis and characterized by UV spectrum, FTIR, XRD. It has good antioxidant activity which is determined by DPPH assay and the total antioxidant activity was determined by Phosbomolybdate assay. So in future the Sterptomyces $s p$. DSK2 melanin silver nanoparticle and the melanin pigment were used pharmaceutical studies and cosmetic preparations, optical and UV protectant such as creams and lotions.

\section{ACKNOWLEDGMENTS}

The authors wish to thank the Dr.G.Arjunan, Head of the Department, Department of Animal Sciences, Bharathidasan University, Tiruchirappalli-24, India, for providing the opportunity and Dr.D.Dhanasekaran, Assistant Professor, Department of Microbiology, Bharathidasan University, Tiruchirappalli. We are thankful to once again for supporting research ideas and providing facilities.

\section{REFERENCES}

[1]. Larsson B.S.: "Pigment Cell Res"- 6, 127 (1993).

[2]. Hong L., Simon J.D.: J. Phys. Chem. B. 111, 7938 (2007). 
[3]. Liu, Y. T., M. J. Sui, D. D. Ji, I. H. Wu, C. Chou, and C. C. Chen. "Protection from UV irradiation by melanin of mosquitocidal activity of Bt. var. Israeliensis”. J. Invertebr. Pathol. 62: 131-136. 1993.

[4]. Larsson BS, "Interaction between chemicals and melanin". Pigment Cell Res 6:127-133.

[5]. Zane P, Brindle SD, Gause D, O'Buck AJ, Raghavan PR, Tripp $\mathrm{SL}$, "Physicochemical factors associated with binding and retention of compounds in ocular melanin of rats: correlations using data from whole-body autoradiography and molecular modeling for multiple linear regression analyses". Pharm Res 7:935-941, 1990

[6]. Atlasik B, Stephen K, Wilczok T , Interaction of drugs with ocular melanin in vitro". Exp Eye Res 30:pp 325-33,1980

[7]. Debing I, Ijzerman AP, Vauqelin G, "Melanosome binding and oxidation-reduction properties of synthetic L-dopa-melanin as in vitro tests for drug toxicity". Mol Pharmacol 33:470-476, 1988

[8]. Shimada K, Baweja R, Sokoloski T, Patil PN, “ Binding characteristics of drugs to synthetic levodopa melanin". J Pharm Sci 65:1057-1060, 1976.

[9]. Buszman E., Wrzeuniok D., Trzcionka J.: Pharmazie 62, 210 ,2007.

[10]. Wrzeuniok D., Buszman E., Karna E., Paka J.: Pharmazie 60, 439 (2005).

[11]. Wrzeuniok D., Buszman E., Lakota D.: Acta Pol. Pharm. Drug Res. 68, 493 (2011).

[12]. M.M. Salazar-Bookaman, I. Wainer, P.N. Patil, J. Ocul. Pharmacol. Vol.10, 217-220,1994.

[13]. N.G. Lindquist, S. Ullberg, Acta Pharmacol. Toxicol (Copenh.) 2(Suppl) 1972

[14]. Nordberg G.F., Fowler B.A., Nordberg M.,Friberg L.: "Handbook on the Toxicology of

[15]. Metals". 3rd edition., pp. 6- 8, Elsevier, Amsterdam 2007.

[16]. Huang J, Chen C, He N, Hong J, Lu Y, Qingbiao L, Shao W, Sun $\mathrm{D}$, Wang XH, Wang Y, Yiang,"Biosynthesis of silver and gold nanoparticles by novel sundried Cinnamomum camphora Leaf". Nanotechnology Issue-18: pp-105-106., 2007.

[17]. Rai M, Yadav A, Gade A, "Current trends in phytosynthesis of metal nanoparticles. Crit Rev

[18]. Biotechnology". 28(4):277-284, 2008

[19]. Husseiny MI, El-Aziz MA, Badr Y, Mahmoud MA,'Biosynthesis of gold nanoparticles

[20]. using Pseudomonas aeruginosa". Spectrochim Acta A: Mol Biomol Spectrosc 67: pp- 1003-1006, 2007.
[21]. Shahverdi AR, Minaeian S, Shahverdi HR, Jamalifar H, Nohi AA, "Rapid synthesis of silver

[22]. nanoparticles using culture supernatants of Enterobacteria: a novel biological approach".

[23]. Process Biochem 42: pp-919-923, 2007.

[24]. Kumar V, Yadav SK,"Plant-mediated synthesis of silver and gold nanoparticles and their

[25]. Applications". J Chem Technol Biotechnol 84:pp- 151-157, 2009.

[26]. Parikh RY, Singh S, Prasad BLV, Patole MS, Sastry M, Shouche YS, "Extracellular synthesis of crystalline silver nanoparticles and molecular evidence of silver resistance from Morganella sp: towards understanding biochemical synthesis mechanism" ChemBioChem .9:pp- 1415-1422, 2008.

[27]. Gajbhiye M, Kesharwani J, Ingle A, Gade A, Rai M, "Fungusmediated synthesis of silver nanoparticles and their activity against pathogenic fungi in combination with fluconazole.Nanomed" Nanotechnol Biol Med vol-5:pp-382386.2009.

[28]. Ahmad A, Senapati S, Khan MI, Kumar R, Ramani R, Shrinivas $\mathrm{V}$, Sastry M,'Intracellular synthesis of gold nanoparticles by a novel alkalotolerant actinomycete, Rhodococcus species".Nanotechnology vol-14:pp-824-828, 2003b.

[29]. Shahi SK, Patra M, "Microbially synthesized bioactive nanoparticles and their formulation active against human pathogenic fungi". Rev Adv Mater Sci 5:501-509, 2003.

[30]. Singaravelu G, Arockiamary JS, Ganesh Kumar V, Govindraju K, "A novel extracellular synthesis of monodisperse gold nanoparticles using marine alga, Sargassum wightii Greville".Colloid Surf B: Biointerface 57: pp-97-101, 2007.

[31]. Chakraborty N, Banerjee A, Lahiri S, Panda A, Ghosh AN, Pal R, "Biorecovery of gold using cyanobacteria and eukaryotic alga with special reference to nanogold formation - a novel phenomenon". J Appl Phycol 21(1):145-152, 2009.

[32]. Gade AK, Bonde P, Ingle AP, Marcato PD, Duran N, Rai MK, "Exploitation of Aspergillus niger for synthesis of silver nanoparticles". J Biobased Mater Bioenergy 2:pp- 243-247, 2008.

[33]. Mukherjee P, Ahmad A, Mandal D, Senapati S, Sainkar SR, Khan MI, Ramani R, Parischa R, Ajayakumar PV, Alam M, Sastry M, Kumar R ,"Bioreduction of AuCl4ions by the fungus Verticillium $s p$ and surface trapping of the gold nanoparticles formed". Angew Chem Int Ed 40(19): pp-3585-3588, 2001.

[34]. J. Gallas, M. Eisner, Melanin polyvinyl alcohol plastic laminates for optical applications, US Patent7029758 , 2006 